\title{
Erratum to: Evaluation of the Effect of NT-3 and Biodegradable Poly-L-lactic Acid Nanofiber Scaffolds on Differentiation of Rat Hair Follicle Stem Cells into Neural Cells In Vitro
}

\author{
Fatemeh Moghani Ghoroghi ${ }^{1}$ - Leila Beygom Hejazian ${ }^{2}$ - Banafshe Esmaeilzade ${ }^{3}$. \\ Masumeh Dodel $^{4} \cdot$ Masoud Roudbari $^{5} \cdot$ Maliheh Nobakht $^{6,7}$
}

Published online: 1 December 2016

(C) Springer Science+Business Media New York 2016

Erratum to: J Mol Neurosci (2013) 51:318-327

DOI 10.1007/s12031-013-0073-x

The original version of this article unfortunately contained mistakes in the author group and affiliation sections. Banafshe Esmaeilzade's name was incorrectly presented as "Banafshe Esmaielzade". Her affiliation should also be corrected to "Anatomy Department, School of Medicine, Bushehr University of Medical Sciences, Bushehr, Iran".

The online version of the original article can be found at http://dx.doi. org/10.1007/s12031-013-0073-x.

Maliheh Nobakht

nobakht@yahoo.com

1 Anatomy Department, School of Medicine, Tehran University of Medical Sciences, Tehran, Iran

2 Anatomy Department, School of Medicine, Babol University of Medical Sciences, Babol, Iran

3 Anatomy Department, School of Medicine, Bushehr University of Medical Sciences, Bushehr, Iran

4 Nanotechnology and Tissue Engineering Department, Stem Cell Technology Research Center, Tehran, Iran

5 Statistics and Mathematics Department, School of Health Management and Information Sciences, Iran University of Medical Sciences, Tehran, Iran

6 Histology \& Neuroscience Department, School of Medicine, Iran University of Medical Sciences, Tehran, Iran

7 Anti-microbial Resistance Research Center, Iran University of Medical Sciences, Hemmat Highway, P.O. Box 14155-6183, Tehran, Iran 